\title{
Sobolev regularity via the convergence rate of convolutions and Jensen's inequality
}

\author{
Mark A. Peletier, Robert Planqué and Matthias RöGer
}

\begin{abstract}
We derive a new criterion for a real-valued function $u$ to be in the Sobolev space $W^{1,2}\left(\mathbb{R}^{n}\right)$. This criterion consists of comparing the value of a functional $\int f(u)$ with the values of the same functional applied to convolutions of $u$ with a Dirac sequence. The difference of these values converges to zero as the convolutions approach $u$, and we prove that the rate of convergence to zero is connected to regularity: $u \in W^{1,2}$ if and only if the convergence is sufficiently fast. We finally apply our criterium to a minimization problem with constraints, where regularity of minimizers cannot be deduced from the Euler-Lagrange equation.
\end{abstract}

Mathematics Subject Classification (2000): 46E35 (primary); 49J45, 49J40 (secondary).

\section{Introduction}

Jensen's inequality states that if $f: \mathbb{R} \rightarrow \mathbb{R}$ is convex and $\varphi \in L^{1}\left(\mathbb{R}^{n}\right)$ with $\varphi \geq 0$ and $\int \varphi=1$, then

$$
\int_{\mathbb{R}^{n}} f(u(x)) \varphi(x) d x \geq f\left(\int_{\mathbb{R}^{n}} u(x) \varphi(x) d x\right),
$$

for any $u: \mathbb{R}^{n} \rightarrow \mathbb{R}$ for which the integrals make sense. A consequence of this inequality is that

$$
\int_{\mathbb{R}^{n}} f(u)=\int_{\mathbb{R}^{n}} f(u) * \varphi \geq \int_{\mathbb{R}^{n}} f(u * \varphi) .
$$

In this paper we investigate the inequality (1.1) more closely. In particular we study the relationship between the regularity of a function $u \in L^{2}\left(\mathbb{R}^{n}\right)$ and the asymptotic

The first and the third author were supported by NWO grant 639.032.306. The second one wishes to thank the Centrum voor Wiskunde en Informatica for their financial support.

Received February 27, 2007; accepted in revised form October 1, 2007. 
behaviour as $\varepsilon \rightarrow 0$ of

$$
T_{f}^{\varepsilon}(u):=\int_{\mathbb{R}^{n}}\left[f(u)-f\left(u * \varphi_{\varepsilon}\right)\right] .
$$

Here $\varphi_{\varepsilon}(y)=\varepsilon^{-n} \varphi\left(\varepsilon^{-1} y\right)$ and $f$ is a smooth function, but now not necessarily convex. Since $u * \varphi_{\varepsilon} \rightarrow u$ almost everywhere, we find for 'well-behaved' $f$ that $T_{f}^{\varepsilon}(u) \rightarrow 0$ as $\varepsilon \rightarrow 0$. Our aim is to establish a connection between the rate of convergence of $T_{f}^{\varepsilon}(u)$ to zero and the regularity of $u$.

Such a connection between the decay rate of $T_{f}^{\varepsilon}(u)$ and the regularity of $u$ is suggested by the following informal arguments. Taking $n=1$, and assuming $\varphi$ to be even and $u$ to be smooth, we develop $u * \varphi_{\varepsilon}$ as

$$
u * \varphi_{\varepsilon}(x)=\int_{\mathbb{R}} u(x-y) \varphi_{\varepsilon}(y) d y \approx u(x)+\frac{\varepsilon^{2}}{2} u^{\prime \prime}(x) \int_{\mathbb{R}} y^{2} \varphi(y) d y,
$$

so that

$$
T_{f}^{\varepsilon}(u) \approx-c \varepsilon^{2} \int_{\mathbb{R}} f^{\prime}(u) u^{\prime \prime}=c \varepsilon^{2} \int_{\mathbb{R}} f^{\prime \prime}(u)\left|u^{\prime}\right|^{2},
$$

with $c=\frac{1}{2} \int_{\mathbb{R}} y^{2} \varphi(y) d y$. This suggests that if $u \in W^{1,2}(\mathbb{R})$ then $T_{f}^{\varepsilon}(u)$ is of order $\varepsilon^{2}$. Moreover, since $f^{\prime \prime} \geq 0$ in the case that $f$ is convex, (1.4) gives an enhanced version of (1.1).

Conversely, for functions not in $W^{1,2}\left(\mathbb{R}^{n}\right)$ we might not observe decay of $T_{f}^{\varepsilon}(u)$ with the rate of $\varepsilon^{2}$, as a simple example shows. Take $f(u)=u^{2}$ and consider a function with a jump singularity such as $u(x)=H(x)-H(x-1) \notin W^{1,2}(\mathbb{R})$, where $H(x)$ is the Heaviside function. Now choose as regularization kernels the functions $\varphi_{\varepsilon}(x)=\frac{1}{2 \varepsilon}(H(x+\varepsilon)-H(x-\varepsilon))$, after which an explicit calculation shows that $T_{f}^{\varepsilon}(u)=2 \varepsilon / 3$. Here the decay is only of order $\varepsilon$.

The goal of this paper is to prove the asymptotic development (1.4) in arbitrary dimension and to show that also the converse statement holds true: if $T_{f}^{\varepsilon}(u)$ is of order $\varepsilon^{2}$ then $u \in W^{1,2}\left(\mathbb{R}^{n}\right)$. These results are stated below and proved in Sections 2 and 3 .

The fact that one can deduce regularity from the decay rate is the original motivation of this work. In Section 4 we illustrate the use of this result with a minimization problem in which the Euler-Lagrange equation provides no regularity for a minimizer $u$. Instead we estimate the decay of $T_{f}^{\varepsilon}(u)$ directly from the minimization property and obtain that $u$ is in $W^{1,2}$.

Our results can be compared to the characterisation of Sobolev spaces introduced by Bourgain, Brezis and Mironescu [2,4]. In fact, the regularity conclusion in Theorem 1.2 could be derived from [2], with about the same amount of effort as the self-contained proof that we give here.

More generally one could try to characterize the class of functions $u$ for which $\varepsilon^{-\alpha} T_{f}^{\varepsilon}(u)$ is controlled uniformly in $\varepsilon>0$. We briefly discuss this question in Section 5 . 


\subsection{Notation and assumptions}

Let $\varphi \in L^{1}\left(\mathbb{R}^{n}\right)$ satisfy

$$
\begin{aligned}
& \varphi \geq 0 \quad \text { in } \mathbb{R}^{n}, \\
& \int_{\mathbb{R}^{n}} \varphi=1, \\
& \int_{\mathbb{R}^{n}} y \varphi(y) d y=0, \\
& \int_{\mathbb{R}^{n}}|y|^{2} \varphi(y) d y<\infty .
\end{aligned}
$$

For $\varepsilon>0$ we define the Dirac sequence

$$
\varphi_{\varepsilon}(x):=\frac{1}{\varepsilon^{n}} \varphi\left(\frac{x}{\varepsilon}\right) .
$$

Eventually we will restrict ourselves to the case that $\varphi$ is rotationally symmetric, that is $\varphi(x)=\tilde{\varphi}(|x|)$, where $\tilde{\varphi}: \mathbb{R}_{0}^{+} \rightarrow \mathbb{R}_{0}^{+}$. Then (1.6) is equivalent to

$$
n \omega_{n} \int_{0}^{\infty} r^{n-1} \tilde{\varphi_{\varepsilon}}(r) d r=1,
$$

where $\omega_{n}$ denotes the volume of the unit-ball, i.e. $\left|B_{1}(0)\right|=\omega_{n}$.

For a function $u \in L^{1}\left(\mathbb{R}^{n}\right)$ and $0 \leq s \leq 1$ we define the convolution $u_{\varepsilon}$ and modified convolutions $u_{\varepsilon, s}$ by

$$
\begin{aligned}
u_{\varepsilon}(x) & :=\left(u * \varphi_{\varepsilon}\right)(x)=\int_{\mathbb{R}^{n}} u(x-y) \varphi_{\varepsilon}(y) d y, \\
u_{\varepsilon, s}(x) & :=\left(u * \varphi_{\varepsilon s}\right)(x)=\int_{\mathbb{R}^{n}} u(x-y) \varphi_{\varepsilon s}(y) d y=\int_{\mathbb{R}^{n}} u(x-s z) \varphi_{\varepsilon}(z) d z .
\end{aligned}
$$

Note that $u_{\varepsilon}=u_{\varepsilon, 1}, u=u_{\varepsilon, 0}$.

We also use the notation $a \otimes b$ for the tensor product of $a, b \in \mathbb{R}^{n}$.

\subsection{Statement of main results}

Our first result proves (1.4).

Theorem 1.1. Let $f \in C^{2}(\mathbb{R})$ have uniformly bounded second derivative and

$$
f(0)=0, \quad f^{\prime}(0)=0 .
$$

Let $\left(\varphi_{\varepsilon}\right)_{\varepsilon>0}$ be a Dirac sequence as in Section 1.1. Then for any $u \in W^{1,2}\left(\mathbb{R}^{n}\right)$,

$$
\lim _{\varepsilon \rightarrow 0} \frac{1}{\varepsilon^{2}} \int_{\mathbb{R}^{n}}\left[f(u)-f\left(u_{\varepsilon}\right)\right] d x=\frac{1}{2} \int_{\mathbb{R}^{n}} f^{\prime \prime}(u(x)) \nabla u(x) \cdot A(\varphi) \nabla u(x) d x,
$$


where

$$
A(\varphi)=\int_{\mathbb{R}^{n}}(y \otimes y) \varphi(y) d y .
$$

If $\varphi$ is rotationally symmetric, i.e. $\varphi(x)=\tilde{\varphi}(|x|)$, then

$$
\lim _{\varepsilon \rightarrow 0} \frac{1}{\varepsilon^{2}} \int_{\mathbb{R}^{n}}\left[f(u)-f\left(u_{\varepsilon}\right)\right] d x=\frac{1}{2} a(\varphi) \int_{\mathbb{R}^{n}} f^{\prime \prime}(u(x))|\nabla u(x)|^{2} d x,
$$

with

$$
a(\varphi)=\omega_{n} \int_{0}^{\infty} r^{n+1} \tilde{\varphi}(r) d r .
$$

The second theorem shows that for uniformly convex $f$ a decay of $T_{f}^{\varepsilon}(u)$ of order $\varepsilon^{2}$ implies that $u \in W^{1,2}\left(\mathbb{R}^{n}\right)$.

Theorem 1.2. Let $f \in C^{2}(\mathbb{R})$ have uniformly bounded second derivative, assume that (1.13) is satisfied, and that there is a positive number $c_{1}>0$ such that

$$
f^{\prime \prime} \geq c_{1} \quad \text { on } \mathbb{R} \text {. }
$$

If for $u \in L^{2}\left(\mathbb{R}^{n}\right)$,

$$
\liminf _{\varepsilon \rightarrow 0} \frac{1}{\varepsilon^{2}} \int_{\mathbb{R}^{n}}\left[f(u(x))-f\left(u_{\varepsilon}(x)\right)\right] d x<\infty,
$$

then $u \in W^{1,2}\left(\mathbb{R}^{n}\right)$. In particular (1.14) and (1.16) hold.

Remark 1.3. We observe that by (1.13), $f(u)$ and $f\left(u_{\varepsilon}\right)$ are integrable. In fact, by the assumptions on $f$ we deduce that

$$
|f(y)| \leq\left\|f^{\prime \prime}\right\|_{\infty}|y|^{2} .
$$

Hence, for all $u \in L^{2}\left(\mathbb{R}^{n}\right)$ we obtain that $|f(u)| \leq\left.|| f^{\prime \prime}\right|_{\infty}|u|^{2} \in L^{1}\left(\mathbb{R}^{n}\right)$.

Since $u_{\varepsilon} \in L^{2}\left(\mathbb{R}^{n}\right)$ for $u \in L^{2}\left(\mathbb{R}^{n}\right)$ we similarly deduce that $f\left(u_{\varepsilon}\right) \in L^{1}\left(\mathbb{R}^{n}\right)$.

For functions $u \in L^{1}\left(\mathbb{R}^{n}\right) \cap L^{2}\left(\mathbb{R}^{n}\right)$ Theorems 1.1 and 1.2 hold even without assuming (1.13).

\section{Proof of Theorem 1.1}

We first compute that

$$
\begin{aligned}
T_{f}^{\varepsilon}(u) & =-\int_{\mathbb{R}^{n}} \int_{0}^{1} \frac{\partial}{\partial s} f\left(u_{\varepsilon, s}(x)\right) d s d x \\
& =\int_{0}^{1} \int_{\mathbb{R}^{n}} \int_{\mathbb{R}^{n}} f^{\prime}\left(u_{\varepsilon, s}(x)\right) \nabla u(x-s y) \cdot y \varphi_{\varepsilon}(y) d y d x d s
\end{aligned}
$$


and that

$$
\begin{aligned}
f^{\prime}\left(u_{\varepsilon, s}(x)\right)-f^{\prime}\left(u_{\varepsilon, s}(x-s y)\right) & =-\int_{0}^{s} \frac{\partial}{\partial r} f^{\prime}\left(u_{\varepsilon, s}(x-r y)\right) d r \\
& =\int_{0}^{s} f^{\prime \prime}\left(u_{\varepsilon, s}(x-r y)\right) \nabla u_{\varepsilon, s}(x-r y) \cdot y d r .
\end{aligned}
$$

Since for all $0 \leq s \leq 1$

$$
\begin{aligned}
\int_{\mathbb{R}^{n}} \int_{\mathbb{R}^{n}} f^{\prime}\left(u_{\varepsilon, s}(x-s y)\right) \nabla u(x-s y) \cdot y \varphi_{\varepsilon}(y) d y d x \\
=\int_{\mathbb{R}^{n}} \int_{\mathbb{R}^{n}} f^{\prime}\left(u_{\varepsilon, s}(z)\right) \nabla u(z) \cdot y \varphi_{\varepsilon}(y) d z d y \\
\stackrel{(1.7)}{=} 0,
\end{aligned}
$$

we deduce from (2.1), (2.2) that

$$
\begin{aligned}
& \frac{1}{\varepsilon^{2}} \int_{\mathbb{R}^{n}}\left[f(u(x))-f\left(u_{\varepsilon}(x)\right)\right] d x \\
= & \frac{1}{\varepsilon^{2}} \int_{0}^{1} \int_{\mathbb{R}^{n}} \int_{\mathbb{R}^{n}}\left[\left(\int_{0}^{s} f^{\prime \prime}\left(u_{\varepsilon, s}(x-r y)\right) \nabla u_{\varepsilon, s}(x-r y) \cdot y d r\right)\right. \\
\left.\nabla u(x-s y) \cdot y \varphi_{\varepsilon}(y)\right] d y d x d s & \frac{1}{\varepsilon^{2}} \int_{0}^{1} \int_{0}^{s} \int_{\mathbb{R}^{n}} \int_{\mathbb{R}^{n}}\left[f^{\prime \prime}\left(u_{\varepsilon, s}(x)\right) \nabla u_{\varepsilon, s}(x) \cdot y\right. \\
= & \frac{1}{\varepsilon^{n+2}} \int_{0}^{1} \int_{0}^{s} \int_{\mathbb{R}^{n}} f^{\prime \prime}\left(u_{\varepsilon, s}(x)\right) \nabla u_{\varepsilon, s}(x) \\
= & \frac{1}{\varepsilon^{n+2}} \int_{0}^{1} \int_{0}^{s} \int_{\mathbb{R}^{n}}(y \otimes y) \cdot \nabla u(x-r y) \varphi\left(\varepsilon^{-1} y\right) d y d x d r d s \\
f_{\mathbb{R}^{n}}^{\prime \prime}\left(u_{\varepsilon, s}(x)\right) \nabla u_{\varepsilon, s}(x) & \int_{0}^{1} \int_{0}^{s} \int_{\mathbb{R}^{n}} f^{\prime \prime}\left(u_{\varepsilon, s}(x)\right) \nabla u_{\varepsilon, s}(x) \cdot\left(\kappa_{\varepsilon r} * \nabla u\right)(x) d x d r d s, \\
= & \quad(z) \cdot \nabla u(x-z) \varphi\left(\varepsilon^{-1} r^{-1} z\right) d z d x d r d s
\end{aligned}
$$

with the modified convolution kernel $\kappa_{\varepsilon r}$ defined by

$$
\kappa_{\varepsilon r}(z):=(\varepsilon r)^{-n} \kappa\left(\frac{z}{\varepsilon r}\right),
$$


where $\kappa$ is given by

$$
\kappa(y):=(y \otimes y) \varphi(y) .
$$

As $\varepsilon \rightarrow 0$ we have that for all $0 \leq r, s \leq 1$,

$$
\begin{aligned}
u_{\varepsilon, s} & \rightarrow u & & \text { almost everywhere, } \\
\nabla u_{\varepsilon, s} & \rightarrow \nabla u & & \text { in } L^{2}\left(\mathbb{R}^{n}\right), \\
\kappa_{\varepsilon r} * \nabla u & \rightarrow\left(\int_{\mathbb{R}^{n}} \kappa(y) d y\right) \nabla u & & \text { in } L^{2}\left(\mathbb{R}^{n}\right) .
\end{aligned}
$$

Moreover, the integrand on the right-hand side of (2.4) is dominated by the function

$$
\frac{1}{2}\left\|f^{\prime \prime}\right\|_{L^{\infty}(\mathbb{R})}\left(\left|\nabla u_{\varepsilon, s}\right|^{2}+\left|\kappa_{\varepsilon r} * \nabla u\right|^{2}\right),
$$

which converges strongly in $L^{1}\left(\mathbb{R}^{n}\right)$ as $\varepsilon \rightarrow 0$ by (2.6) and (2.7). We therefore deduce from (2.4) that

$$
\begin{aligned}
& \lim _{\varepsilon \rightarrow 0} \frac{1}{\varepsilon^{2}} \int_{\mathbb{R}^{n}}\left[f(u(x))-f\left(u_{\varepsilon}(x)\right)\right] d x \\
= & \int_{0}^{1} \int_{0}^{s} \int_{\mathbb{R}^{n}} f^{\prime \prime}(u(x)) \nabla u(x) \cdot\left(\int_{\mathbb{R}^{n}} \kappa(y) d y\right) \nabla u(x) d x d r d s \\
= & \frac{1}{2} \int_{\mathbb{R}^{n}} f^{\prime \prime}(u(x)) \nabla u(x) \cdot\left(\int_{\mathbb{R}^{n}} \kappa(y) d y\right) \nabla u(x) d x .
\end{aligned}
$$

In the rotationally symmetric case we observe that

$$
\begin{aligned}
\int_{\mathbb{R}^{n}}(y \otimes y) \varphi(y) d y & =\left(\int_{0}^{\infty} r^{n+1} \tilde{\varphi}(r) d r\right) \int_{S^{n-1}} \theta \otimes \theta d \theta \\
& =\omega_{n}\left(\int_{0}^{\infty} r^{n+1} \tilde{\varphi}(r) d r\right) I d .
\end{aligned}
$$

Here we used that for $i, j=1, \ldots, n$,

$$
\int_{S^{n-1}} \theta_{i} \theta_{j} d \theta= \begin{cases}\omega_{n} & \text { if } i=j \\ 0 & \text { if } i \neq j\end{cases}
$$

We therefore obtain from (2.4)-(2.10) that in the rotationally symmetric case

$$
\lim _{\varepsilon \rightarrow 0} \frac{1}{\varepsilon^{2}} \int_{\mathbb{R}^{n}}\left[f(u)-f\left(u_{\varepsilon}\right)\right] d x=\omega_{n}\left(\int_{0}^{\infty} r^{n+1} \tilde{\varphi}(r) d r\right) \frac{1}{2} \int_{\mathbb{R}^{n}} f^{\prime \prime}(u)|\nabla u|^{2} d x .
$$




\section{Proof of Theorem 1.2}

Let

$$
\Lambda:=\liminf _{\varepsilon \rightarrow 0} \frac{1}{\varepsilon^{2}} \int_{\mathbb{R}^{n}}\left[f(u(x))-f\left(u_{\varepsilon}(x)\right)\right] d x .
$$

We first remark that by assumption (1.18) the function $r \mapsto f(r)-\frac{c_{1}}{2} r^{2}$ is convex and we deduce again by (1.1) that

$$
\int_{\mathbb{R}^{n}}\left[\left(f(u)-\frac{c_{1}}{2} u^{2}\right)-\left(f\left(u_{\varepsilon}\right)-\frac{c_{1}}{2} u_{\varepsilon}^{2}\right)\right] \geq 0 .
$$

This implies that

$$
\liminf _{\varepsilon \rightarrow 0} \frac{1}{\varepsilon^{2}} \int_{\mathbb{R}^{n}}\left(u^{2}-u_{\varepsilon}^{2}\right) \leq \frac{2}{c_{1}} \liminf _{\varepsilon \rightarrow 0} \frac{1}{\varepsilon^{2}} \int_{\mathbb{R}^{n}}\left[f(u)-f\left(u_{\varepsilon}\right)\right]=\frac{2}{c_{1}} \Lambda .
$$

Next we consider $\delta>0$ and the regularizations $u_{\delta}=u * \varphi_{\delta}$. Set $\gamma_{\varepsilon}:=\varphi_{\varepsilon} * \varphi_{\varepsilon}$. Then we obtain that

$$
\begin{aligned}
& \int_{\mathbb{R}^{n}}\left[u_{\delta}^{2}(x)-\left(u_{\delta} * \varphi_{\varepsilon}\right)^{2}(x)\right] d x \\
= & \int_{\mathbb{R}^{n}} u_{\delta}^{2}-\int_{\mathbb{R}^{n}} \int_{\mathbb{R}^{n}} u_{\delta}(x) u_{\delta}(y) \gamma_{\varepsilon}(x-y) d y d x \\
= & \frac{1}{2} \int_{\mathbb{R}^{n}} \int_{\mathbb{R}^{n}}\left(u_{\delta}(x)-u_{\delta}(y)\right)^{2} \gamma_{\varepsilon}(x-y) d y d x \\
\stackrel{(1.1)}{\leq} & \frac{1}{2} \int_{\mathbb{R}^{n}} \int_{\mathbb{R}^{n}} \int_{\mathbb{R}^{n}}(u(x-z)-u(y-z))^{2} \varphi_{\delta}(z) d z \gamma_{\varepsilon}(x-y) d y d x \\
= & \frac{1}{2} \int_{\mathbb{R}^{n}} \int_{\mathbb{R}^{n}} \int_{\mathbb{R}^{n}}(u(\xi)-u(\eta))^{2} \gamma_{\varepsilon}(\xi-\eta) d \eta d \xi \varphi_{\delta}(z) d z \\
= & \frac{1}{2} \int_{\mathbb{R}^{n}} \int_{\mathbb{R}^{n}}(u(\xi)-u(\eta))^{2} \gamma_{\varepsilon}(\xi-\eta) d \eta d \xi \\
= & \int_{\mathbb{R}^{n}}\left[u^{2}(\xi)-u_{\varepsilon}^{2}(\xi)\right] d \xi .
\end{aligned}
$$

We therefore deduce from Theorem 1.1 and (3.3), (3.4) that

$$
\begin{aligned}
\int_{\mathbb{R}^{n}}\left|\nabla u_{\delta}\right|^{2} & =C(\varphi) \liminf _{\varepsilon \rightarrow 0} \frac{1}{\varepsilon^{2}} \int_{\mathbb{R}^{n}}\left[u_{\delta}^{2}(x)-\left(u_{\delta} * \varphi_{\varepsilon}\right)^{2}(x)\right] d x \\
& \leq C(\varphi) \liminf _{\varepsilon \rightarrow 0} \frac{1}{\varepsilon^{2}} \int_{\mathbb{R}^{n}}\left[u^{2}(x)-u_{\varepsilon}^{2}(x)\right] d x \leq \frac{2}{c_{1}} \Lambda C(\varphi) .
\end{aligned}
$$

Since this estimate is uniform in $\delta>0$ it follows that $u \in W^{1,2}\left(\mathbb{R}^{n}\right)$. By Theorem 1.1 we therefore deduce (1.14) and (1.16). 


\section{Application: regularity of minimizers in a lipid bilayer model}

We illustrate the utility of Theorems 1.1 and 1.2 with an example. The problem is to determine the regularity of solutions of the following minimization problem.

Problem 4.1. Let $\alpha>0, h>0$ and a kernel $\kappa \in W^{1,1}\left(\mathbb{R}, \mathbb{R}_{0}^{+}\right)$be given such that $\kappa^{\prime} \in B V(\mathbb{R})$. Denote by $\tau_{h}$ the translation operator,

$$
\left(\tau_{h} u\right)(x):=u(x-h) \quad \text { for } u: \mathbb{R} \rightarrow \mathbb{R} .
$$

Consider the set $K \subset \mathbb{R}$,

$$
K:=\left\{u \in L^{1}(\mathbb{R}) \mid \int_{\mathbb{R}} u=1, u \geq 0, u+\tau_{h} u \leq 1 \text { a.e. }\right\}
$$

and a strictly convex, increasing, and smooth function $f$ with $f(0)=f^{\prime}(0)=0$. We then define a functional $F: K \rightarrow \mathbb{R}$ by

$$
F(u):=\int_{\mathbb{R}} f(u)-\int_{\mathbb{R}} \alpha u \kappa * u
$$

and consider the problem of finding a function $u \in K$ that minimizes $F$ in $K$, i.e.

$$
F(u)=\min \{F(v) \mid v \in K\} .
$$

Remark 4.2. This problem arises in the modelling of lipid bilayers, biological membranes (see [1] for more details). There, a specific type of molecules is considered, consisting of two beads connected by a rigid rod. The beads have a certain volume, but the rod occupies no space.

The beads are assumed to be of sub-continuum size, but the rod length is nonnegligeable at the continuum scale. In the one-dimensional case we also assume that the rods lie parallel to the single spatial axis. Combining these assumptions we model the distribution of such molecules over the real line by a variable $u$ that represents the volume fraction occupied by leftmost beads. The volume fraction of rightmost beads is then given by $\tau_{h} u$, and in the condition $u+\tau_{h} u \leq 1$ we now recognize a volume constraint.

The functional $F$ represents a free energy. In the entropy term $\int f(u)$ the function $f$ is strictly convex, increasing, and smooth and can be assumed to satisfy $f(0)=f^{\prime}(0)=0$. The destabilizing term $-\int u \kappa * u$ is a highly stylized representation of the hydrophobic effect, which favours clustering of beads.

Without the constraints $u \geq 0$ and $u+\tau_{h} u \leq 1$ present in (4.1), we would immediately be able to infer that minimizers (even stationary points) are smooth using a simple bootstrap argument: since $u \in K$ we have $u \in L^{p}(\mathbb{R})$ and $\kappa * u \in$ $W^{1, p}$ for all $1 \leq p \leq \infty$, and therefore, using the Euler-Lagrange equation,

$$
f^{\prime}(u)-2 \alpha \kappa * u=\lambda,
$$


for some $\lambda \in \mathbb{R}$, we find $u \in W^{1, p}(\mathbb{R})$. Iterating this procedure we obtain that $u \in$ $W^{k, p}(\mathbb{R})$ for all $k \in \mathbb{N}, 1 \leq p \leq \infty$. However, when including the two constraints, two additional Lagrange multipliers $\mu$ and $v$ appear in the Euler-Lagrange equation,

$$
f^{\prime}(u)-2 \alpha \kappa * u=\lambda+\mu-v-\tau_{-h} \nu .
$$

Here $\mu$ and $v$ are measures on $\mathbb{R}$, and standard theory provides no further regularity than this. In this case the lack of regularity in the right-hand side interferes with the bootstrap process, and this equation therefore does not give rise to any additional regularity.

The interest of Theorem 1.2 for this case lies in the fact that $K$ is closed under convolution. In particular, if $u$ is a minimizer of $F$ in $K$, then $u_{\varepsilon}=\varphi_{\varepsilon} * u$ is also admissible, and we can compare $F\left(u_{\varepsilon}\right)$ with $F(u)$. From this comparison and an application of Theorem 1.2 we deduce the regularity of $u$ :

Corollary 4.3. Let $u$ be a minimizer of (4.3). Then $u \in W^{1,2}(\mathbb{R})$.

Proof. Choose a function $\varphi \in L^{1}(\mathbb{R})$ as in Section 1.1 with Dirac sequence $\left(\varphi_{\varepsilon}\right)_{\varepsilon>0}$, and set $u_{\varepsilon}:=\varphi_{\varepsilon} * u$. First we show that there exists a constant $C \in \mathbb{R}$ such that for all $u \in L^{2}(\mathbb{R})$

$$
\left|\int_{\mathbb{R}}\left(u \kappa * u-u_{\varepsilon} \kappa * u_{\varepsilon}\right)\right| \leq C \varepsilon^{2} \int_{\mathbb{R}} u^{2} .
$$

With this aim we observe that

$$
\int_{\mathbb{R}}\left(u \kappa * u-u_{\varepsilon} \kappa * u_{\varepsilon}\right)=\int_{\mathbb{R}} u\left(\kappa * u-\kappa * u * \gamma_{\varepsilon}\right)
$$

with

$$
\gamma:=\varphi * \varphi, \quad \gamma_{\varepsilon}(x):=\left(\varphi_{\varepsilon} * \varphi_{\varepsilon}\right)(x)=\frac{1}{\varepsilon} \gamma\left(\frac{x}{\varepsilon}\right),
$$

and that $\gamma, \gamma_{\varepsilon}$ satisfy the assumptions in Section 1.1.

Since $\kappa^{\prime} \in B V(\mathbb{R})$ we have $(\kappa * u)^{\prime \prime}=\kappa^{\prime \prime} * u \in L^{2}(\mathbb{R})$ and

$$
\left\|\kappa^{\prime \prime} * u\right\|_{L^{2}(\mathbb{R})} \leq\left(\int_{\mathbb{R}}\left|\kappa^{\prime \prime}\right|\right)\|u\|_{L^{2}(\mathbb{R})} .
$$

Repeating some arguments of Theorem 1.1 we calculate that

$$
\begin{aligned}
(\kappa * u)(x)-\left(\kappa * u * \gamma_{\varepsilon}\right)(x) & =-\int_{0}^{1} \frac{d}{d s} \int_{\mathbb{R}}(\kappa * u)(x-s y) \gamma_{\varepsilon}(y) d y d s \\
& =\int_{0}^{1} \int_{\mathbb{R}}(\kappa * u)^{\prime}(x-s y) y \gamma_{\varepsilon}(y) d y d s \\
& =\int_{0}^{1} \int_{0}^{1} \int_{\mathbb{R}}(\kappa * u)^{\prime \prime}(x-r s y) s y^{2} \gamma_{\varepsilon}(y) d y d s d r \\
& =\varepsilon^{2} \int_{0}^{1} \int_{0}^{1} \int_{\mathbb{R}}(\kappa * u)^{\prime \prime}(x-\varepsilon r s z) s z^{2} \gamma(z) d z d r d s
\end{aligned}
$$


and therefore that

$$
\begin{aligned}
& \left|\int_{\mathbb{R}} u\left(\kappa * u-\kappa * u * \gamma_{\varepsilon}\right)\right| \\
\leq & \varepsilon^{2} \int_{0}^{1} \int_{0}^{1} \int_{\mathbb{R}}\left[\int_{\mathbb{R}}(\kappa * u)^{\prime \prime}(x-\varepsilon r s z)^{2} d x\right]^{\frac{1}{2}}\left[\int_{\mathbb{R}} u(x)^{2} d x\right]^{\frac{1}{2}} s z^{2} \gamma(z) d z d r d s \\
\leq & \varepsilon^{2} C(\gamma)\left\|\kappa^{\prime \prime} * u\right\|_{L^{2}(\mathbb{R})}\|u\|_{L^{2}(\mathbb{R})} \\
\leq & \varepsilon^{2} C(\varphi, \kappa)\|u\|_{L^{2}(\mathbb{R})}^{2},
\end{aligned}
$$

which by (4.5) implies (4.4).

Since $K$ is closed under convolution with $\varphi_{\varepsilon}, u_{\varepsilon}$ is admissible, and therefore

$$
0 \leq F\left(u_{\varepsilon}\right)-F(u) \leq \int_{\mathbb{R}}\left[f\left(u_{\varepsilon}\right)-f(u)\right]+C \varepsilon^{2} \int_{\mathbb{R}} u^{2} .
$$

The last term is bounded by

$$
C \varepsilon^{2}\|u\|_{L^{1}(\mathbb{R})}\|u\|_{L^{\infty}(\mathbb{R})} \leq C \varepsilon^{2},
$$

which follows from combining both inequality constraints in (4.1).

Therefore $\int\left[f(u)-f\left(u_{\varepsilon}\right)\right]=O\left(\varepsilon^{2}\right)$, and from Theorem 1.2 we conclude that $u \in W^{1,2}(\mathbb{R})$.

As is described in more detail in the thesis [3], Corollory 4.3 paves the way towards rigorously deriving the Euler-Lagrange equations for Problem (4.3), and forms an important ingredient of the proof of existence of minimizers.

\section{Other decay rates}

Here we briefly touch the question how to characterize the class of functions $u$ for which $\varepsilon^{-\alpha} T_{f}^{\varepsilon}(u)$ is controlled uniformly in $\varepsilon>0$. The results of this paper give a complete answer for $\alpha=2$. The example in the introduction shows that for $\alpha=1$ one cannot expect more regularity than that of the Heaviside-function. For $1<\alpha<2$ the following calculation suggests that a control on $\varepsilon^{-\alpha} T_{f}^{\varepsilon}(u)$ is related to the $W^{\alpha / 2,2}\left(\mathbb{R}^{n}\right)$-regularity of $u$.

Proposition 5.1. Let $f(u)=u^{2}, 1<\alpha<2$, and assume that $u \in W^{\alpha / 2,2}\left(\mathbb{R}^{n}\right)$. Then

$$
\lim _{\varepsilon \rightarrow 0} \frac{1}{\varepsilon^{\alpha}} T_{f}^{\varepsilon}(u)=0 .
$$


Proof. We first use Plancherel's Theorem to compute that

$$
\begin{aligned}
T_{f}^{\varepsilon}(u) & =\int_{\mathbb{R}^{n}}\left[u^{2}-\left(u * \varphi_{\varepsilon}\right)^{2}\right] \\
& =\frac{1}{2 \pi} \int_{\mathbb{R}^{n}}\left[|\hat{u}|^{2}-\left|\widehat{u * \varphi_{\varepsilon}}\right|^{2}\right] \\
& =\frac{1}{2 \pi} \int_{\mathbb{R}^{n}}|\hat{u}(\xi)|^{2}\left(1-|\hat{\varphi}(\varepsilon \xi)|^{2}\right) d \xi,
\end{aligned}
$$

where for a function $g$ we denote by $\hat{g}(\xi)=\int_{\mathbb{R}^{n}} e^{-i \xi \cdot x} g(x) d x$ its Fourier transform. We further compute, using (1.6), (1.7) that

$$
\begin{aligned}
1-|\hat{\varphi}(\varepsilon \xi)|^{2} & =1-\left(\int_{\mathbb{R}^{n}} e^{-i \varepsilon \xi \cdot x} \varphi(x) d x\right)\left(\int_{\mathbb{R}^{n}} e^{i \varepsilon \xi \cdot z} \varphi(z) d z\right) \\
& =1-\int_{\mathbb{R}^{n}} \int_{\mathbb{R}^{n}} e^{-i \varepsilon \xi \cdot(x-z)} \varphi(x) \varphi(z) d x d z \\
& =\int_{\mathbb{R}^{n}} \int_{\mathbb{R}^{n}}\left(1-e^{-i \varepsilon \xi \cdot(x-z)}\right) \varphi(x) \varphi(z) d x d z \\
& =\int_{\mathbb{R}^{n}} \int_{\mathbb{R}^{n}}(1-\cos (\varepsilon \xi \cdot(x-z))) \varphi(x) \varphi(z) d x d z .
\end{aligned}
$$

Together with (5.2) this implies that

$$
\varepsilon^{-\alpha} T_{f}^{\varepsilon}(u)=\int_{\mathbb{R}^{n}} \int_{\mathbb{R}^{n}} \int_{\mathbb{R}^{n}}|\hat{u}(\xi)|^{2}|\xi \cdot(x-z)|^{\alpha} \varphi(x) \varphi(z) g(\varepsilon \xi \cdot(x-z)) d x d z d \xi,
$$

where

$$
g(r):=\frac{1-\cos r}{|r|^{\alpha}}
$$

Next we observe that $g$ is uniformly bounded on $\mathbb{R}$ and that $g(r) \rightarrow 0$ as $r \rightarrow 0$. Using (1.8), (5.3), and $u \in W^{\alpha / 2,2}\left(\mathbb{R}^{n}\right)$ we now deduce (5.1) from Lebesgue's Dominated Convergence theorem. 


\section{References}

[1] J. G. Blom and M. A. Peletier, A continuum model of lipid bilayers, European J. Appl. Math. 15 (2004), 487-508.

[2] J. Bourgain, H. Brezis and P. Mironescu, Another look at Sobolev spaces, In: "Optimal Control and Partial Differential Equations" José Luis, Menaldi et. al. (eds.), Amsterdam, IOS Press; Tokyo, Ohmsha, 2001, 439-455.

[3] R. Planqué, "Constraints in Applied Mathematics: Rods, Membranes, and Cuckoos", Ph.D. thesis, Technische Universiteit Delft, 2005.

[4] A. C. Ponce, A new approach to Sobolev spaces and connections to $\Gamma$-convergence, Calc. Var. Partial Differential Equations 19 (2004), 229-255.

Technische Universiteit Eindhoven Den Dolech 2

P.O. Box 513

$5600 \mathrm{MB}$ Eindhoven

m.a.peletier@tue.nl

Department of Mathematics

Vrije Universiteit

De Boelelaan 1081a

1081 HV Amsterdam

rplanque@few.vu.nl

Max Planck Institute

for Mathematics in the Sciences

Inselstr. 22

D-04103 Leipzig

roeger@mis.mpg.de 